J Urol Nephrol

Short Communication 2019 Vol.:6, Issue:1

(c) All rights are reserved by Adapa S, et al.

\title{
Renal Failure from Cast Nephropathy: Role of Plasmapheresis
}

\section{Short Communication}

In multiple myeloma renal involvement is common, occurring in $50 \%$ of the patients and is an independent predictor of poor patient survival [1]. These circulating paraproteins can cause a variety of renal manifestations depending on the site of involvement, type and load of protein involved and mechanism of injury inflicted, resulting in glomerular diseases, proximal tubulopathy and obstructive nephropathy [2].

Light chain cast nephropathy is one of the common renal manifestations, resulting from circulating plasma free light chains. Improved renal recovery has been associated with early reduction of light chains. The evidence on the role of Plasmapheresis combined with chemotherapy alleviating renal failure by reducing the light chains load is conflicting. It does lower serum free light chains rapidly and might reduce renal toxicity, but its affects on patient survival and recovery of renal function by itself yet to be demonstrated [5].

In a systematic review of literature by Kumar et al. concluded that overall survival as well as the need for dialysis is not affected by plasmapheresis in multiple myeloma patients with acute renal failure [3]. Meta analysis by Alkhatib et al. suggested plasmapheresis might be beneficial in cast nephropathy patients proven by biopsy in combination with chemotherapy. This Meta analysis included 147 patients with more than $50 \%$ of them receiving plasmapheresis and treatment schedule varied from 4 to 7 sessions. Plasmapheresis group has lower risk of dialysis dependence compared to the control group but no overall difference in mortality risk at 6 months. There are ongoing clinical trials to assess high-cutoff dialysis as a promising alternative. Most of the clinical trials reported were before the introduction of proteasome inhibitors and immunomodulators which improved response, complete remission, progression free and overall survival compared to conventional chemotherapeutic agents [4]. Another systematic review in patients with acute renal failure from multiple myeloma reported no benefit of plasmapheresis in renal function improvement or overall survival. In this meta analysis, 8 articles met the inclusion criteria out of which 3 were Randomized Controlled Clinical Trials (RCTs). Two out of these 3 RCTs showed majority of patients attained dialysis independence, but showed no difference in survival. All studies demonstrated that the patients receiving plasmapheresis showed an improvement in renal function, however statistically significant improvement in renal function was proven only in 2 RCTs and one retrospective study [6].

Burnette et al. presented their experience of 14 patients over 6.5 yrs receiving proteasome inhibitor [bortezomib] and serial plasma exchange within a week of diagnosis of cast nephropathy. They reported $43 \%$ of the patients with complete and partial renal response respectively. Combined use of bortezomib and plasmapheresis is effective in improving the acute renal failure in patients with multiple
Journal of

Urology \& Nephrology

\section{Konala VM ${ }^{1}$ and Adapa $\mathrm{S}^{2 *}$}

${ }^{1}$ Department of Internal Medicine, Division of Medical Oncology, USA

${ }^{2}$ Division of Nephrology, USA

Address for Correspondence

Adapa S, The Nephrology group 568 East Herndon Avenue \#201, Fresno, CA 93720, Phone: 5592286600, Fax: 5592263709; E-mail: sreedharadapa@gmail.com

Submission: 17 January, 2019

Accepted: 20 February, 2019

Published: 22 February, 2019

Copyright: (c) 2018 Konala VM, et al. This is an open access article distributed under the Creative Commons Attribution License, which permits unrestricted use, distribution, and reproduction in any medium, provided the original work is properly cited.

myeloma presumed due to cast nephropathy [7].

In a study reported by Hutchison et al on multiple patients with severe renal failure and biopsy proven myeloma kidney, demonstrated that early reduction of serum free light chains correlated with improvement in renal function as well as improvement in median survival [8]. In another study reported by the same author involving 67 patients with multiple myeloma causing dialysis dependent renal failure [ $86 \%$ of them with cast nephropathy] was treated with highcutoff dialysis in combination with chemotherapy with approximately two-thirds of the patients achieved independence from dialysis [9].

It is unclear whether reduction of the levels of free light chains represents the efficacy of proteasome inhibitors rather than the efficacy of plasmapheresis.

Two randomized clinical trials (MYRE and the EULITE) assigned patients with biopsy proven cast nephropathy causing acute renal failure with primary end point of discontinuation of renal replacement therapy to combination of proteasome inhibitor-based chemotherapy with either high Cut-Off Hemodialysis (HCO-HD) or High-Flux Hemodialysis (HF-HD). No difference in renal recovery was found in EuLITE study. Patient who were in HCO-HD were independent of dialysis at 6 months as per MYRE study. Both studies were small, which makes any firm conclusions on the benefit of HCO$\mathrm{HD}$ questionable. Based on the current knowledge, without more robust data, the routine use of HCO-HD cannot be recommended [10].

Generally, plasmapheresis is combined with chemotherapy to achieve a $50-60 \%$ reduction in serum free light chains. If dialysis is required on the same day, it is done after the plasmapheresis $[8,11]$.

As per American Society for Apheresis 2016, indications for therapeutic apheresis and cytapheresis procedures-plasmapheresis for renal failure from cast nephropahty in multiple myeloma falls under category 2 recommendation (Disorders for which plasmapheresis for renal failure from cast nephropahty in multiple myeloma is accepted as second-line therapy, either as a standalone treatment or in conjunction with other therapies) and evidence $2 b$ 
(Weak recommendation based on moderate quality evidence) [12].

Most of the clinical trials demonstrating the role of plasmapheresis were done before multiple combination therapies of proteasome inhibitors, immunomodulators, monoclonal antibodies which have significantly altered the management of myeloma with many patients achieving complete remission as well as significant improvement in progression free survival, and overall survival compared to conventional chemotherapy. Plasmapheresis might still be beneficial in a group of patients with cast nephropathy causing renal impairment in combination with chemotherapy. High-cutoff dialysis being another promising alternative to plasmapheresis.

\section{References}

1. Motwani SS, Herlitz L, Monga D, Jhaveri KD, Lam AQ (2016) ParaproteinRelated Kidney Disease: Glomerular Diseases Associated with Paraproteinemias. Clin J Am Soc Nephrol 11: 2260-2272.

2. Nasr SH, Valeri AM, Sethi S, Fidler ME, Cornell LD, et al. (2012) Clinicopathologic correlations in multiple myeloma: a case series of 190 patients with kidney biopsies. Am J Kidney Dis 59: 786-794.

3. Kumar A, Djulbegovic B, Soares HP (2006) Plasmapheresis in the Treatment of Renal Failure Associated with Multiple Myeloma. Am Soc Hematology 3585-3585.

4. Yaser A, Dadla A, Malik D, Abdel-Rahman Z, et al. (2017) Plasmapheresis and Myeloma Cast Nephropathy: A meta-analysis and review of evidence. Am Soc Hematology 5415-5415.
5. Madore F (2015) Plasmapheresis in cast nephropathy: yes or no? Current opinion in nephrology and hypertension 2: 177-182.

6. Gupta D, Bachegowda L, Phadke G, Boren S, Johnson D, et al. (2010) Role of plasmapheresis in the management of myeloma kidney: a systematic review. Hemodial Int 4: 355-363.

7. Burnette BL, Leung N, Raj kumar SV (2011) Renal improvement in myeloma with bortezomib plus plasma exchange. New Eng J Med 24: 2365-2366.

8. Hutchison CA, Cockwell P, Stringer S, Bradwell A, Cook M, et al. (2011) Early reduction of serum-free light chains associates with renal recovery in myeloma kidney. J Am Soc Nephrol 6: 1129-1136.

9. Hutchison CA, Heyne N, Airia P, Schindler R, Zickler D, et al. (2012) Immunoglobulin free light chain levels and recovery from myeloma kidney on treatment with chemotherapy and high cut-off haemodialysis. Nephrol Dial Transplant 10: 3823-3828.

10. Finkel K, Fabbrini P (2017) High cut-off hemodialysis for myeloma cast nephropathy-do we finally have an answer? J Onco-Nephrology 2: 67-70.

11. Hutchison CA, Bradwell AR, Cook M, Basnayake K, Basu S, et al (2009) Treatment of acute renal failure secondary to multiple myeloma with chemotherapy and extended high cut-off hemodialysis. Clin J Am Soc Nephrol 4: 745-754.

12. Schwartz J, Padmanabhan A, Aqui N, Balogun RA, Connelly-Smith L, et al. (2016) Guidelines on the use of therapeutic apheresis in clinical practiceevidence-based approach from the Writing Committee of the American Society for Apheresis: The seventh special issue. J Clin Apher 3: 149-162. 